\title{
Verification of an algorithm for actin tail detection of subviral particles by comparison with expert validated data
}

\begin{abstract}
The locomotion of subviral particles of Marburg virus has been shown to be primarily actin based. For this work, a virologist selected 14 subviral particles that show actin tails in fluorescence image sequences. Using the tracked coordinates, examination areas around these subviral particles are defined. The brightness of within the examination area behind the subviral particle is analysed. In addition, the speed of the particle in each frame is calculated to investigate potential correlations between actin activity and particle speed. The results show that actin tracks can be automatically detected and analysed. First hints of a correlation between subviral particle movement and actin activity could be gathered with the presented actin tail quantifier.
\end{abstract}

Keywords: Actin, Actin tails, subviral particles, image processing

https://doi.org/10.1515/cdbme-2021-2030

\section{Introduction}

It is known that the locomotion of subviral particles of Marburg virus is primarily actin-based [1]. To understand the mechanisms of subviral particle movement, an in-depth analysis of the relation between actin activity distribution in a cell and subviral particle movement is imperative.

A labeling of subviral particles with green fluorescent protein (GFP) and actin structures with red fluorescent protein (RFP) has been performed by virologists. The resulting image sequences serve as basis for the suggested approach. Algorithms to automatically detect and track subviral particles in these sequences have been presented previously [2]. Basing

\footnotetext{
*Corresponding author: Jan Chowanietz: Institute for Biomedical Engineering (IBMT), Faculty of Life Science Engineering (LSE), Technische Hochschule Mittelhessen (THM) University of Applied Sciences, Gießen, Germany, email: jan.chowanietz@Ise.thm.de Andreas Rausch and Thomas Schanze: Technische Hochschule Mittelhessen, Gießen, Germany
}

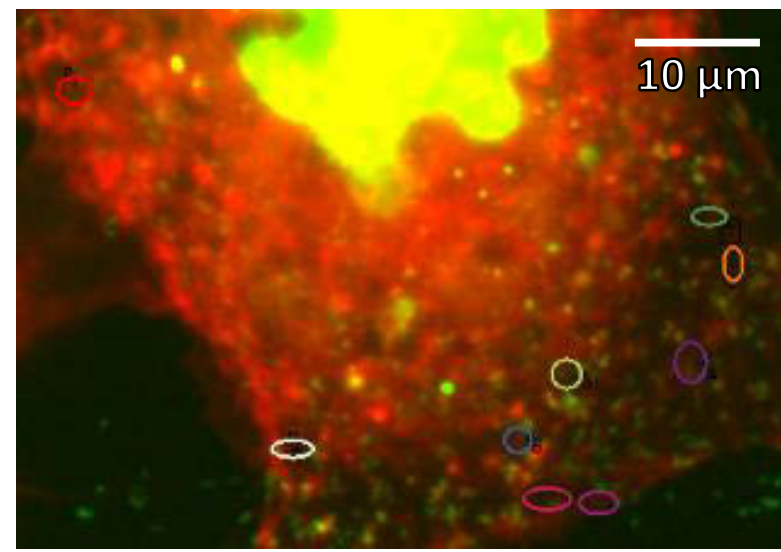

Figure 1: Red and green channel of a section from a fluorescence live cell image. The position of interest that are preselected by a virologist are marked with circles.

on the tracking data, the subviral particle motion can be classified [3]. This allows a detailed study of the influence of actin on the locomotion of subviral particles in cells. Previous work has shown the detectability of actin tails behind moving subviral particles [4].

An algorithm is based on an earlier work by Chowanietz et al. [4], in which actin tail detection of Marburg virus subviral particles in fluorescence microscopy image sequences is presented. This work focuses on the evaluation of the suggested actin tail quantifier with test data, preselected by a virologist of the Institute of Virology at the University of Marburg.

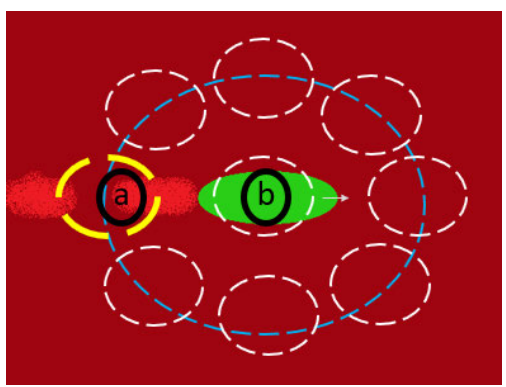

Figure 2: Schematic representation of the examination areas around a subviral particle. (a) Yellow marked examination area behind the particle. (b) Subviral particle. Note the cloud-like bright red actin tail. White examination areas around the subviral particle are discussed in Section 4. 


\section{Material and methods}

\subsection{Validation data}

Fluorescence microscopic image sequences of Marburg virusinfected live cells provided by the Institute of Virology, Philipps-University, Marburg, form the basis of this work. The Leica DMI6000B microscope with a $63 x$ oil objective was used for this purpose. The images were aquired with the Leica LAS software. For qualitative testing, 14 subviral particles with significant brightness intensity changes in their actin tails have been selected by a virologist. The locations of the high actin activity have been registered. Nine positions are labelled as shown in Figure 1. The shown labels mark a rough local position of the high actin activities. Furthermore, the activities extend over multiple frames of the image sequence. The actin tail of the subviral particles of Marburg viruses behave globularly rather than filamentously. Thus, the manual detections serve as approximative tempo-spatial locations for the algorithm validation.

\subsection{Actin tail detection and subviral particle speed}

The algorithm by Chowanietz et al. [4] is briefly presented: The algorithm determines the intensity of the actin tails of each preselected subviral particle by measuring the brightness of the red channel directly behind the subviral particle. However, it is assumed that high brightness correlates with high actin activity. The location of the examination area is illustrated in Figure 2. The intensity is determined for each subviral particle position along the respective track. For the measurement, the orientation of the subviral particle is considered. It is calculated from the current and the last position of the subviral particle. A mean value is then calculated from each of the nine examination areas around the subviral particle (Figure 2). This is repeated for each image. The results are stored in an array. The speed of each subviral particle is calculated for each frame according to Chowanietz et al. [4].

\subsection{Comparison of the detected actin tails}

Diagrams are created for the comparison of the manual expertdetections of actin tails with the algorithmic results. For this purpose, the frame area where an actin tail was seen by the virologist is marked in the diagrams. A red box highlights the manual expert-detections in the diagrams (Figure 3). A qualitative visual comparison is made possible by examining the results only in the area of the red box. The remaining course of actin brightness over time is used to correctly characterize the actin brightness intensity.

\section{Results}

Fourteen subviral particle tracks, classified by a virologist, were examined. Three examples are given for the presentation of results. In the first subsection, the results to verify the actin tail detection algorithm are presented. In the second subchapter, the results for the investigation of the correlation between speed and actin brightness are presented.

Table 1: The table shows the results of the evaluation for the 14 subviral particles. Assignment of particle track numbers to manually as well as automatically indexed actin tail frame numbers. A "no statement possible" is entered if it cannot be clearly determined. A "-" is entered if no actin tail is detected.

\begin{tabular}{ccc}
\hline $\begin{array}{c}\text { Track } \\
\text { number }\end{array}$ & $\begin{array}{c}\text { Frame number of } \\
\text { manually detected } \\
\text { actin tail }\end{array}$ & $\begin{array}{c}\text { Frame numbers of the } \\
\text { automatically detected } \\
\text { actin tail }\end{array}$ \\
\hline 1 & 30 & $25-35$ \\
2 & 60 & - \\
3 & 80 & $80-90$ \\
4 & 45 & no statement possible \\
5 & 8 & $50-60$ \\
6 & 54 & $70-85$ \\
7 & 86 & - \\
8 & 87 & $120-140$ \\
9 & 132 & $50-60$ \\
10 & 57 & 180 \\
11 & 180 & $95-105$ \\
12 & 103 & - \\
13 & 93 & $75-90$ \\
14 & 93 &
\end{tabular}

\subsection{Actin brightness}

The manually detected events of high actin activities are compared with the automatically detected actin tail intensity profiles. Subviral particles number 6,11 , and 13 were selected as examples from Table 1 and shown in Figure 3.

Figure $3 \mathrm{a}$ presents the result for subviral particle number 6 . The $\mathrm{X}$-axis indicates the frames of the fluorescence image 
(a)

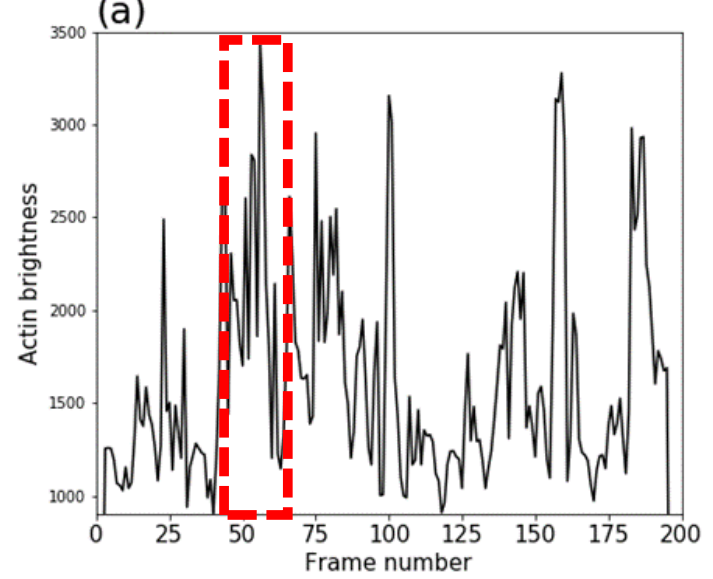

(b)

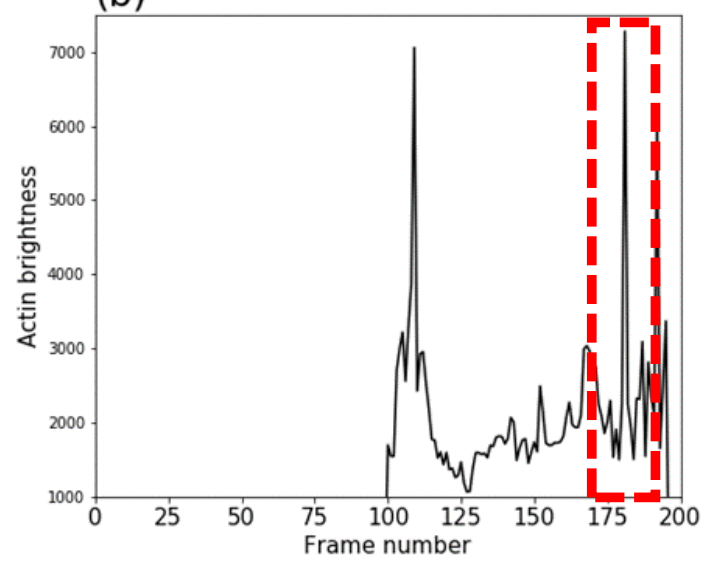

(c)

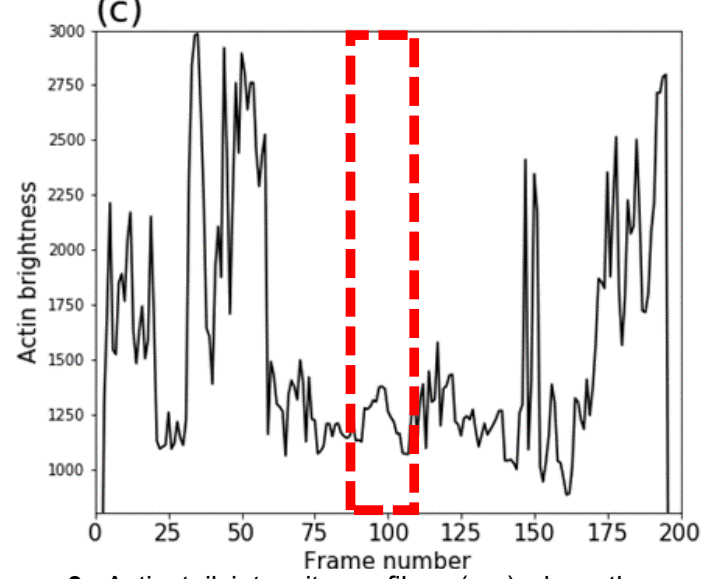

Figure 3: Actin tail intensity profiles. (a-c) show the measured intensities of the actin tails in each frame from the respective subviral particles, listed in Table 1, (a) Subviral particle number 6, (b) subviral particle number 11 and (c) subviral particle number 13.

sequence. The Y-axis shows the actin brightness. The virologist saw an actin tail at frame 54. Therefore, the red box, which marks the temporal region where the virologist saw an actin tail, is placed between frame 50 and frame 60. The algorithm detects an increased actin brightness value around the same area. This is consistent with the virologist's statement. Figure 4 shows the actin tail that was detected by the virologist.

For subviral particle number 11, the time course of the actin brightness is shown in Figure 3b. According to the virologist, an actin tail can be detected in the 180th frame region. A pronounced peak of actin intensity is located at frame 180, which means within the manual detection marked by a red box. Figure $3 \mathrm{c}$ shows the result for subviral particle number 13 . The virologist saw an actin tail in the 93rd frame region. This is marked by the red box. No significant deflection of actin brightness was registered in this region by the algorithm. The actin brightness intensity is in the normal range compared to, for example, frame 50 . This therefore contradicts the statement of the virologist.

Table 1 shows a success rate of $75 \%$ for matching for a match between frame numbers of the manually and the automatically detected actin tails.

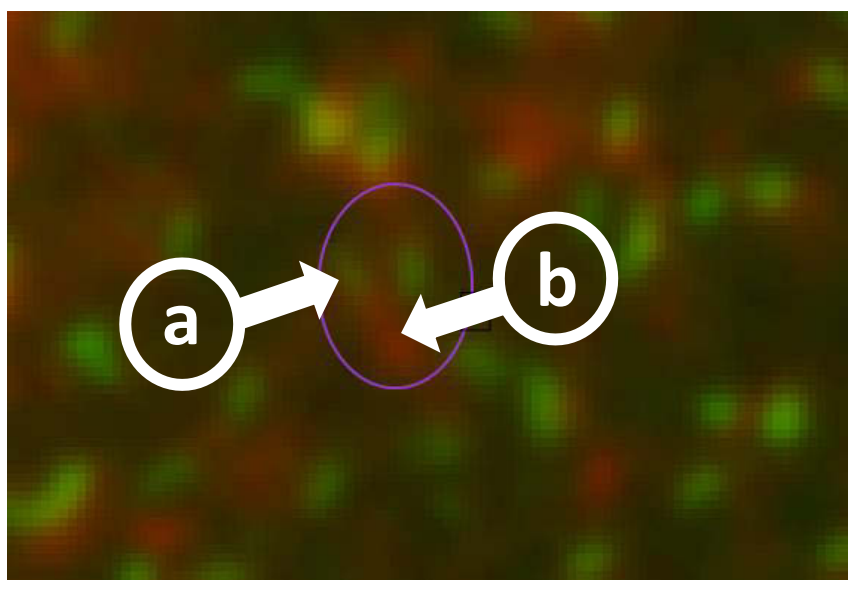

Figure 4: Subviral particle with actin tail. Section of the fluorescence image sequence showing an actin tail behind a subviral particle. (a) Subviral particle number 6. (b) Actin tail.

\subsection{Relationship between particle speed and actin tail brightness}

The relation between actin tail brightness and subviral particle speed is investigated exemplarily investigted on subviral particle number 11 . Figure 5 shows the measured speed and actin tail intensity profile of the exemplary subviral particle. The left $\mathrm{Y}$-axis shows the speed in $\mathrm{nm} / \mathrm{s}$. The right $\mathrm{Y}$-axis shows the actin brightness in the examination area behind the particle. The $\mathrm{X}$-axis shows the frame number of the fluorescence image sequence. The measurements are visually investigated for distinct correlations. A distinct actin tail activity followed by a rapid increase of the subviral particle's speed is present between frame number 100 and frame number 125 . 


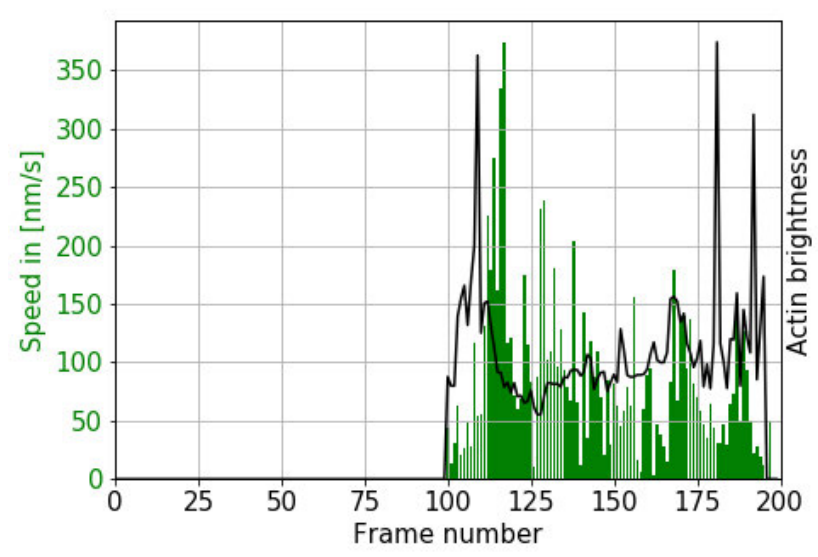

Figure 5: Actin tail intensity profile (black) in relation to the speed (green) of the subviral particle number 11 of Marburg virus.

\section{Discussion and Conclusions}

The main aim of this work is the verification of the initial work of Chowanietz et al. [2]. In collaboration with the Institute of Virology at the University of Marburg, 14 subviral particles with an actin tail were selected for the verification. The manually detected results were compared with the automatically detected results. Nine $(75 \%)$ of the manually detected high actin activities have been also detected by the presented algorithm.

Subviral particle number 11 (Figure 3b) is used as a prime example. It distinctly shows a match between manual and automated detection of high actin tail activity. However, such uniqueness is rare. Mostly, smaller peaks as in Figure 3a occur repeatedly. For three subviral particles, no agreement of actin tail and actin brightness intensity could be made. Figure 3c serves as an example. No actin brightness increase was measured in the area of the red box.

From the 14 subviral particles, no conclusion could be reached in two cases, because the particle tracks were erroneously terminated prematurely by the tracking algorithm and partially continued as a new track. The random separation of the tracks can lead to a loss of information and thus worsen actin tail detection. In this case, the consequence was that the track of the subviral particle was too short and too few reference values existed to make a conclusion about whether there was an increase in actin brightness intensity in this region. The two particles were removed from the evaluation. Future work should aim for more stable tracking.

The reason for the discontinuation was the crossing of two particle tracks, a problem that also affects actin tail detection.
Figure 4 shows that actin tail detection is difficult to implement due to a high density of subviral particles. In the example shown, two particles move almost side by side for a short time. Crossing of actin tails may lead to temporal artificial increases of the intensity due to the superimposition. To compensate the effect of actin tail superimposition, a spatio-temporal averaging of the image data should be investigated in future work.

The purpose of detecting the actin tail is to better investigate the interaction between subviral particles and actin. Figure 5 is a first example and more data needs to be collected. It shows, it is also possible in future work to analyze possible relations, e. g. correlation or even causality, between actin tail activity and subviral particle velocity. In future work it should also be considered to evaluate the actin signal in examination areas around the subviral particle, as illustrated in Figure 2.

The presented actin tail quantifier shows promising potential for the analysis of subviral particle movement and actin activity recorded by fluorescence microscopy imaging of virus-infected cells.

\section{Acknowledgments}

We want to thank the work group of Stephan Becker from the Institute for Virology, Marburg, Germany. Especially, we want to thank Olga Dolnik for providing fluorescence microscopic image sequences for our research.

\section{Author Statement}

Research funding: The author states no funding involved. Conflict of interest: Authors state no conflict of interest. Informed consent: Informed consent has been obtained from all individuals included in this study.

\section{References}

[1] G. Schudt, L. Kolesnikova, O. Dolnik, B. Sodeik, S. Becker, Transport of Marburg virus nucleocapsids, Proceedings of the National Academy of Sciences, Aug 2013.

[2] A. Rausch, D. Müller, T. Schanze, Improvement of a subviral particle tracker by the use of a LAP-Kalman-algorithm. Current Directions in Biomedical Engineering, 2(1), 2016, pp. 415-418.

[3] A. Rausch, T. Schanze, Fractal Dimensions of Subviral Particle Movement, Current Directions in Biomedical Engineering, vol. 4, no. 1, 2018, pp. 79-82.

[4] J. Chowanietz, A. Rausch, T. Schanze, Actin tail detection in fluorescence microscopic image sequences to investigate the cause of movement of subviral particle, Automed 2021 